\title{
Stratégie globale de réduction des risques d'inondation en Loire moyenne - démarche intégrée de prévention du plan Loire
}

\author{
A global strategy for reducing flood risks in the medium Loire valley
}

par A. Bachoc, R. Thépot, Y. Mérillon, J. Lefèvre, N-G. Camp'huis

(Etat, EPALA, Agence de l'eau Loire Bretagne, Equipe pluridisciplinaire plan Loire grandeur nature)

In order to ensure flood control in the Loire valley, riparians of the river have built $600 \mathrm{~km}$ of dikes. But many times in the past, the river has had huge floods which destroyed $25 \%$ of the dikes and flooded 300000 ha. So the people do accept to let the flood come into the flood-prone area to reduce the power of the stream. The protection is reinforced with a first dam builded in 1984. Because of the 300000 inhabitants and 14000 companies settled in the floodprone area and the cost of the damage evaluated 7 billions USS, the river basin authorities decided to define a strategy to reduce the damages of the flood. A multipurpose study is being completed. It indicates the need of mitigation to reduce damage. Mitigation needs to restore a particular way of life, a culture of life in the flood-prone area and also to define a special program of intervention to predict the flood, to inform the local authorities and to manage the crises during the flood and after it.

\section{I $\square$ UN FLEUVE IMPÉTUEUX ET AMÉNAGÉ DEPUIS PLUSIEURS SIÈCLES}

1.1. Des crues fortes et courtes, d'origine orageuse

La Loire moyenne s'étend sur $430 \mathrm{~km}$ entre le Bec d'Allier et Montjean-sur-Loire. Elle commence à la confluence de la Loire (18 $000 \mathrm{~km}^{2}$ de bassin versant) et de l'Allier (14000 km² de bassin versant).

On ne compte aucun apport majeur de débit en crue entre le Bec d'Allier et le Bec de Cher, soit sur près de $300 \mathrm{~km}$. En aval de Tours, la Loire conflue successivement avec le Cher $\left(14000 \mathrm{~km}^{2}\right)$, l'Indre et la Vienne $\left(21000 \mathrm{~km}^{2}\right)$ en $35 \mathrm{~km}$ puis avec la Maine $\left(22000 \mathrm{~km}^{2}\right) 60 \mathrm{~km}$ plus en aval.

En amont de Tours, les crues rares de la Loire moyenne sont très fortes et brutales, car elles proviennent d'une crue simultanée de la Loire et de l'Allier découlant d'un même orage de grande importance, qui s'étend sur 3 à 4 jours. A titre d'exemple, il est tombé sur l'ensemble des bassins de la Loire et de l'Allier une moyenne de $120 \mathrm{~mm}$ de pluie (soit $20 \%$ de la pluviométrie annuelle) en 4 jours lors de la crue de 1866. Et la moitié a entièrement ruisselé pour se retrouver dans la rivière.

Les crues représentent donc des volumes impressionnants, variant de 1 à 3 milliards de $\mathrm{m}^{3}$, soit une inondation moyenne de 1 à $3 \mathrm{~m}$ si on devait épandre la crue instantanément sur le lit majeur. Or le maximum du volume est concentré dans les quelque douze heures que dure le passage de la pointe de la crue. La rapidité de la crue et de la décrue est un phénomène connu et typique, qui pose de réels problèmes en matière d'alerte des populations et de risque sur la stabilité des ouvrages.

\subsection{Des enjeux importants et particulièrement vulnérables}

Jusqu'à l'avènement du chemin de fer, la Loire a représenté la voie de communication par excellence pour relier l'Atlantique et la Méditerranée avec Paris. Son cours a été corseté pour toujours mieux faciliter la navigation, ce qui était un vrai défi quand on sait que le débit descendait régulièrement en dessous de $50 \mathrm{~m}^{3} / \mathrm{s}$ plusieurs semaines voire un à trois mois par an.

Aujourd'hui, la Loire moyenne n'est pas de ces fleuves défigurés par les aménagements industriels, en partie à cause de ses étiages longtemps trop sévères et contraignants. Il n'empêche que les 33 vals que la crue de 1856 a entièrement inondés sous des hauteurs de 1 à $4 \mathrm{~m}$ d'eau, abritent 300000 personnes dans 250 communes différentes, soit 10 fois plus qu'au siècle dernier. 13600 entreprises sont actuellement implantées en zone inondable, dont 13100 ont moins de 20 salariés. Ces implantations humaines et économiques relativement récentes n'ont jamais eu l'expérience d'une crue, dans ce secteur où la dernière crue cinquantennale en amont de la Vienne est passée en 1907. On dénombre aussi 1550 équipements publics inondables. Tous ces enjeux 


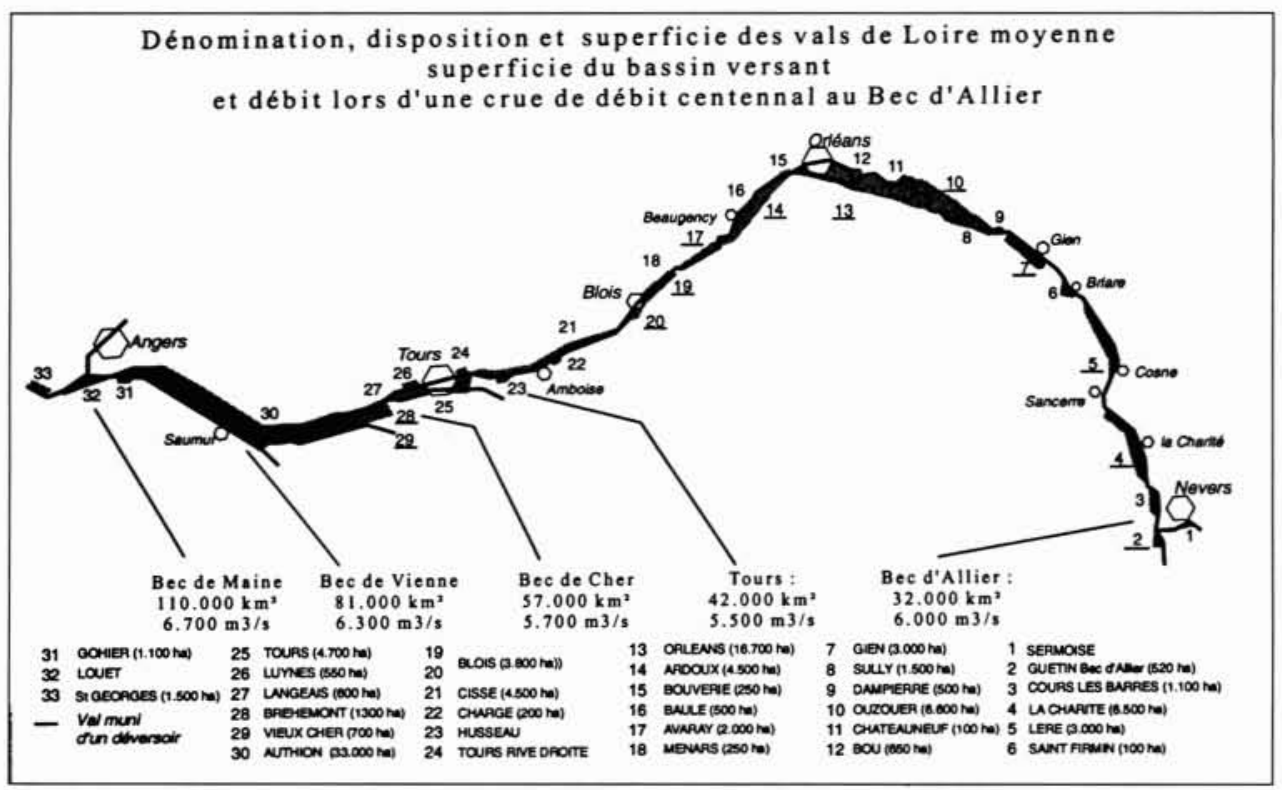

1. Dénomination, disposition et superficie des vals de Loire moyenne -

Superficie du bassin versant et débit lors d'une crue de débit centennal au Bec d'Allier. représentent $15 \%$ de la population et $20 \%$ des entreprises des "zones d'emplois" bordant la Loire.

75 à $80 \%$ des enjeux sont concentrés dans quatre vals (Tours, qui en abrite le tiers, Authion et Orléans le cinquième, Cisse). Il reste 20 à $25 \%$ des habitants et des entreprises disséminés sur le reste du cours dans un grand nombre de communes de faible importance, qui rencontrent souvent des difficultés pour maintenir, en l'absence de crue, un développement économique robuste face aux contraintes actuelles.

Les activités économiques représentent $60 \%$ des dommages de crue évaluables et l'habitat le quart.

\section{II $\square$ UNE PROTECTION COMBINANT DES LEVÉES ET UN BARRAGE ÉCRÊTEUR}

\subsection{Une protection par levées et déversoir de sécurité}

La Loire moyenne est un fleuve aménagé de très longue date. Sur les $430 \mathrm{~km}, 100000$ ha soit les $2 / 3$ du lit du fleuve, sont soustraits aux crues par un système de $550 \mathrm{~km}$ de levées, qui isolent 33 vals d'une longueur variant de 5 à $70 \mathrm{~km}$ et d'une largeur de 1 à $8 \mathrm{~km}$ (cf. figure 1). Les hommes n'ont laissé souvent que $300 \mathrm{~m}$ de largeur à la Loire au droit des ponts anciens, et ailleurs moins de $1 \mathrm{~km}$,

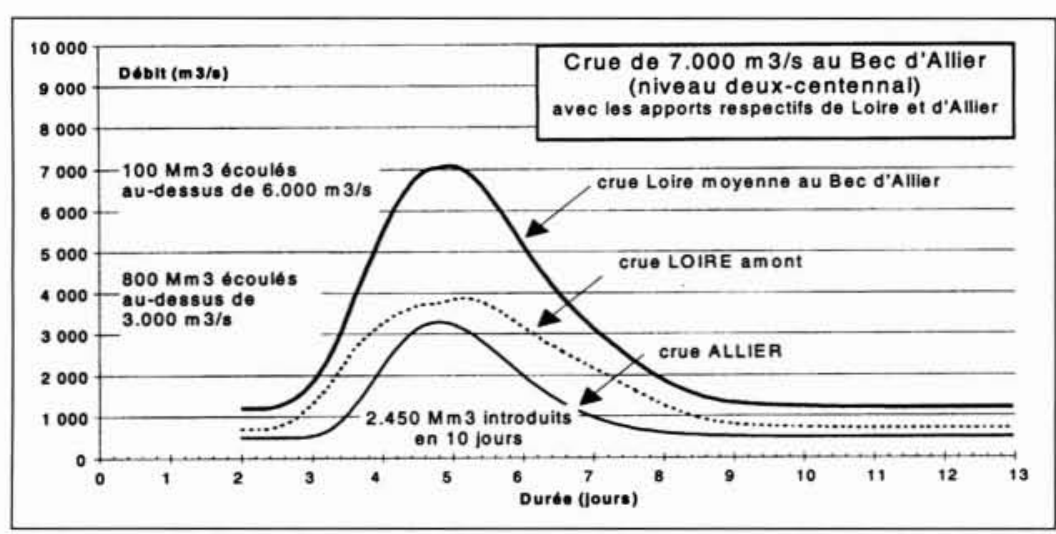

2. Allure des hydrogrammes-types utilisés dans l'étude de modélisation.

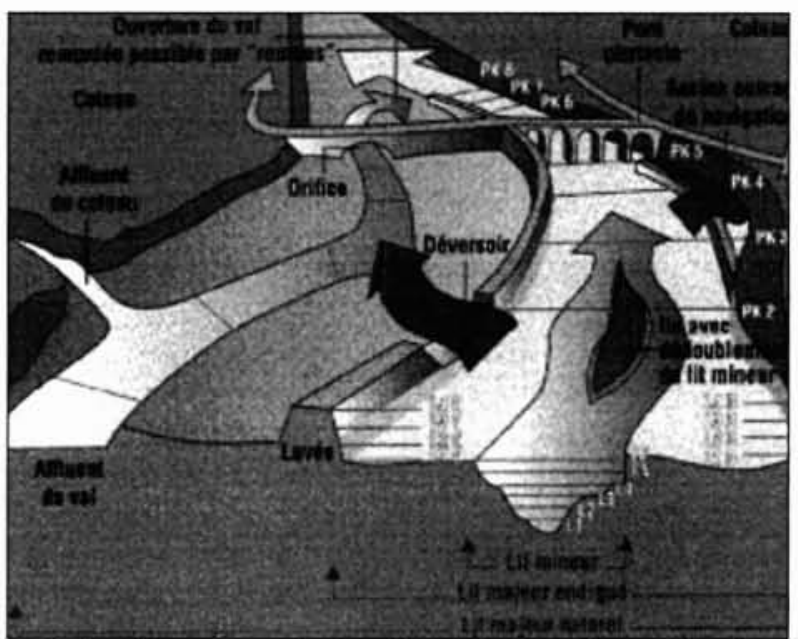

3. Fonctionnement hydraulique de la Loire endiguée.

dans une vallée de 3 à $8 \mathrm{~km}$ de large. Ce système très ancien a systématiquement été surélevé à chaque fois qu'une crue le dépassait. Jusqu'au siècle dernier, les hommes ont cru qu'en surélevant toujours on arriverait à maintenir la Loire entre les digues en terre (cf. figures 3 et 5 ).

Or dès avant la crue centennale, les levées ne protègent plus certains vals car la Loire peut les submerger (cf. figure 3). Au siècle dernier, trois crues d'importance centennale en 20 ans ont ouvert chacune 160 brèches dans les levées, totalisant plus de $100 \mathrm{~km}$ de levées détruites. Les Ligériens ont enfin compris qu'il fallait arrêter de remonter les levées et les ingénieurs de Loire ont proposé de "faire la part de l'eau", en créant des déversoirs de sécurité dans les digues. Ils assurent à l'eau une entrée en un site identifié et protégé (figures 3 et 4 ).

Dans l'esprit de l'époque, il fallait empêcher l'intrusion subite de l'eau dans les vals, consécutive à l'ouverture d'une brèche dans la levée. La technique dérive des "déchargeoirs" testés au XVIII ${ }^{\circ}$ siècle, et consiste à ménager dans la levée un abaissement de la 


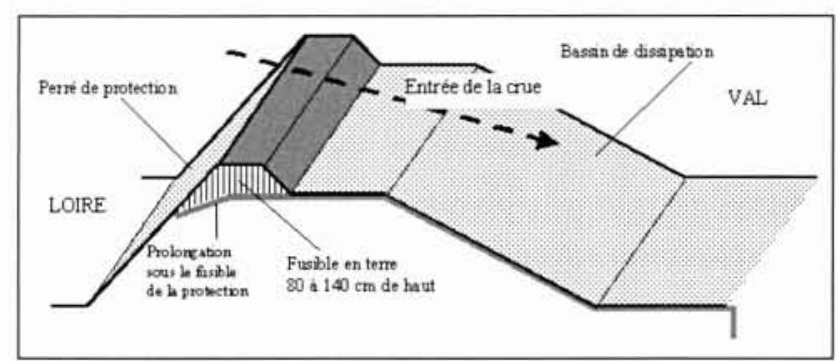

4. Fusible sur un déversoir.

crête en le protégeant par un perré. L'eau débouche dans le val par une sorte de canal aménagé et protégé spécialement.

Le système a été amélioré en ajoutant un "fusible", un bourrelet en terre de 0,8 à $1,6 \mathrm{~m}$ de haut, 30 à $50 \mathrm{~cm}$ de largeur en crête (Figure 4). Il doit résister à la crue jusqu'à ce que l'eau le submerge et en érode la partie en terre enherbée. Il sera alors détruit en un temps censé être bref. Un mur d'eau entrera subitement dans le val, entraînant un fort écrêtement de la crue en Loire. Jusqu'à $1000 \mathrm{~m}^{3} / \mathrm{s}$ entrent dans un val par un déversoir de 500 à $800 \mathrm{~m}$ de long. Si le val derrière le déversoir est resté jusque-là à l'abri de l'inondation, le volume entrant se perd vite. Si son volume est important, l'onde de crue dans le val peut s'étendre au point de revenir s'appuyer sur les levées du côté du val. Elle assure ainsi une certaine protection de ces ouvrages, en équilibrant la poussée côté fleuve. Il ressort rarement plus de 300 à $400 \mathrm{~m}^{3} / \mathrm{s}$ en Loire, avec un décalage marqué avec la pointe de la crue, ce qui écrête fortement la crue.

Sur les 20 déversoirs prévus au siècle dernier, ouvrant aux crues 18 vals et 44000 ha, dans des sites réputés avoir subi des brèches à chaque crue forte, 8 seulement ont été réalisés entre 1870 et 1891 (cf. figure 1). S'y ajoutent trois déversoirs existant déjà avant les crues de 1846 à 1856 (Gien, Ardoux et Blois) et les déversoirs au terrain naturel de Passy, Léré et Gohier.

\subsection{Une évolution récente du lit change le risque de rupture}

Les levées présentent quatre risques de rupture :

- la surverse,

- le renard ou circulation d'eau dans la levée ou sa fondation, qui finit par la déstabiliser,

- l'érosion et la déstabilisation du pied de la levée en contact avec le lit mineur profond,

- la décrue rapide qui déstabilise la levée du côté de la Loire.

Depuis 1970, un important renforcement des levées a diminué le risque de renard dans la levée. Il reste des points de faiblesse : des murets en terre ou maçonnerie ajoutés au siècle dernier au sommet des levées pour éviter toutes les surverses et dont les fondations sont faibles, l'absence de barrière étanche dans les fondations pour lutter contre les renards souterrains, les maisons, canalisations ou autres éléments présents dans le corps des levées et surtout le contact du lit mineur avec le pied de levée.

En effet, une importante campagne d'extraction a retiré entre 1960 et 1990 près de 200 millions de tonnes de granulats de la Loire. Le lit mineur s'est enfoncé en moyenne de 1 à $2 \mathrm{~m}$. Cela accroît le risque de déstabilisation des $120 \mathrm{~km}$ de pieds de levée qui se trouvent au contact direct du lit mineur.

Pour recenser et suivre tout ce qui peut être source de perturbation en période de crue, une reconnaissance de terrain a observé l'état de la levée et de ses protections tous les $500 \mathrm{~m}$. Un système d'information géographique archive toutes ces informations et permet de caractériser les risques, de les hiérarchiser et de recenser les sites où plusieurs risques se trouvent superposés.

\subsection{Un barrage écrêteur existant et un autre ouvrage à l'étude}

Dès le siècle dernier, la construction de barrages dans le haut-bassin pour retenir une partie de la crue a fait partie des moyens étudiés pour diminuer les crues. A l'époque, on avait recensé pas moins de 80 sites de barrages. Réaliser autant d'ouvrages est très coûteux en investissement et en entretien et pose le problème d'une correcte coordination de leur impact, lors de la crue, si on ne veut pas risquer d'aggraver la situation en favorisant la concomitance des maximums des crues de différents affluents.

Le barrage de Villerest, situé sur la Loire, contrôle un bassin versant de $6500 \mathrm{~km}^{2}$, soit $5 \%$ du total. Il peut stocker de l'ordre de $140 \mathrm{Mm}^{3}$ pour écrêter les crues, ce qui lui permet d'abaisser le débit de pointe d'une crue de l'ordre de $1000 \mathrm{~m}^{3 / \mathrm{s}}$. Dans ce cas, il permet d'abaisser le niveau d'eau en crue de l'ordre de $1 \mathrm{~m}$ à
5. Coupe d'une levée type en haut avant renforcement de 1970 (source: Histoire des levées, de Dion) et en bas après renforcement de 1970. 
$20 \mathrm{~cm}$ tout le long de la Loire en aval. Il n'intervient que si la crue entrant dans le barrage dépasse $1600 \mathrm{~m}^{3} / \mathrm{s}$. Mis en fonctionnement en 1985, il n'a fonctionné qu'une seule fois, en novembre 1996, pour écrêter une crue de la Loire.

Pour compléter l'action de Villerest, il conviendrait d'intervenir sur les crues de l'Allier. Seul un site, celui du Veurdre, assure un prélèvement efficace sur les crues. Il est situé sur l'Allier à $25 \mathrm{~km}$ en amont de la confluence avec la Loire, ce qui permettrait de bien voir la crue se former à la fois sur l'Allier et sur la Loire avant de le faire fonctionner. Cet ouvrage, encore en projet, est original, puisqu'il s'agirait d'un barrage à pertuis toujours ouvert, sauf en période de crue. En dehors de la période d'écrêtement d'une crue, à savoir quelques jours tous les 5 à 10 ans, les pertuis ouverts ne retiennent pas l'eau. L'ouvrage n'influence donc pas le fonctionnement de l'Allier. La "retenue" du barrage est vide et laissée à son activité agricole. Les pertuis ne seraient fermés que si le débit de la Loire et de l'Allier ensemble dépassait $3000 \mathrm{~m}^{3} / \mathrm{s}$ au bec d'Allier.

\section{III a UN DIAGNOSTIC DU FONCTIONNE- MENT DU SYSTÈME DE PROTECTION}

\subsection{Une capacité de simuler le fonctionnement et le dysfonctionnement}

Une modélisation de la Loire moyenne mise au point par le bureau d'études Hydratec (France) a permis de disposer d'une image de la Loire en crue, de savoir comment fonctionnerait aujourd'hui le système de protection, d'en détecter les dysfonctionnements et de rechercher des remèdes et des améliorations.

Le modèle décrit la Loire sur $450 \mathrm{~km}$ avec un profil en travers tous les kilomètres, relevés sur le terrain en 1995. Sa mise au point a coûté de l'ordre de $2,5 \mathrm{MF}$ et son utilisation pour faire le diagnostic de la Loire et rechercher des solutions d'amélioration, autant.

Ce modèle a pu être très correctement calé sur des mesures de hauteur d'eau réalisées tous les 1,5 à $2 \mathrm{~km}$, lors de trois crues récentes (1992 à 1996) d'une période de retour comprise entre 2 et 8 ans. On considère que la précision absolue du modèle est de l'ordre de 15 à $25 \mathrm{~cm}$ pour une quelconque des crues simulées. Quand on compare entre eux deux scénarios, les différences sont connues avec une précision de quelques centimètres.

\subsection{Un système de protection par levée toujours très vulnérable}

Le modèle a révélé que les levées et déversoirs actuels n'apportent pas la protection totale que beaucoup de Ligériens leur attribuent inconsciemment.

Des vals de Loire sont fortement inondés (plus de $1 \mathrm{~m}$ d'eau en moyenne) pour des crues inférieures à la centennale, concernant 20000 habitants et près de 1600 entreprises, pour un dommage évalué de l'ordre de 3 à $5 \mathrm{Mds} F$ ( 0,5 à $0,8 \mathrm{M}$. Euros).

La crue atteint le sommet de la levée et vient soit s'appuyer sur la banquette installée en 1846, soit verser pardessus la levée, provoquant alors sa destruction progressive. Le modèle intègre le niveau des levées et permet donc de localiser précisément les endroits concernés, ainsi que les moments de la crue où se pose le problème. Grâce à lui, on peut estimer que le problème concerne $20 \mathrm{~km}$ de banquettes et $6 \mathrm{~km}$ de surverse en crue centennale et $40 \mathrm{~km}$ de banquettes et $18 \mathrm{~km}$ de surverse en crue deux-centennale. Cette information est importante pour évaluer et localiser les travaux éventuels et pour cibler les lieux où une surveillance et une intervention s'imposent en période de crue.

Le lit s'étant enfoncé de manière différente le long de la Loire, les déversoirs de sécurité ne fonctionnent plus exactement à la cote idéale : soit les fusibles rompent pour une crue plus fréquente que prévu, ce qui inonde le val de manière précoce et peu efficace pour l'écrêtement de la crue ; soit ils rompent pour une crue plus rare et laissent passer alors en Loire un débit fort qui peut provoquer des surverses en aval. C'est le cas dans le val d'Orléans, où le déversoir de Jargeau ne fonctionnant plus, l'eau passe par-dessus la levée à l'abord du centre ville, ce qui rend l'inondation catastrophique. Le modèle révèle aussi qu'en donnant des cotes fixes aux fusibles, on n'exploite pas, de manière dynamique, le volume d'écrêtement que représentent les vals. La technique actuellement utilisée pour les barrages n'est pas utilisée pour les vals, car elle conduirait à voir leur inondation programmée et gérée par une autorité et non pas par la seule Loire.

La modélisation révèle que le risque de renard dans les fondations des levées ou de déstabilisation du pied des levées situées au contact direct avec le lit mineur est fortement aggravé. La Loire dispose en effet d'une capacité de remobiliser une épaisseur de 4 à $8 \mathrm{~m}$ de sédiments au fond de son lit mineur pendant la durée de la crue, ce qui peut doubler à décupler la profondeur actuelle de ce lit. Ce phénomène connu a été évalué pour la première fois. Ses conséquences sont préoccupantes, d'autant que pas moins de $130 \mathrm{~km}$ de levées (sur $550 \mathrm{~km}$ ) sont concernés, sur 120 sites différents, tout le long de la Loire.

Or ce sont les brèches accidentelles, en particulier dues à la déstabilisation des levées, qui peuvent augmenter les dommages de manière colossale. En effet, en l'absence de telles brèches, les dommages évaluables sont estimés entre $7 \mathrm{MdF}$ en crue centennale et $17 \mathrm{MdF}$ en crue cinqcentennale. Une seule brèche accidentelle, par exemple dans l'agglomération de Tours ou Orléans, peut augmenter les dommages de près de 7 à $15 \mathrm{MdF}$. Si tous les vals de Loire moyenne se retrouvaient sous l'eau, suite à une série de brèches accidentelles, le dommage dépasserait $40 \mathrm{MdF}$.

L'amélioration est indéniable, mais elle s'accompagne d'une fragilisation supplémentaire des pieds de levée et d'une propension plus forte de la végétation à encombrer le lit et à augmenter les niveaux d'eau en crue.

Le bénéfice montré par un modèle qui suppose le lit bien entretenu et des pieds de levée stables pourrait s'avérer illusoire en l'absence d'un entretien régulier du lit et des levées de la Loire. Le même modèle révèle en effet qu'un lit mal entretenu et dégradé entraîne des surélévations locales de la ligne d'eau de 30 à $70 \mathrm{~cm}$ : un mauvais entretien du lit annihile tous les bénéfices obtenus par la réalisation de Villerest ou le renforcement des levées.

\section{UNE NOUVELLE APPROCHE STRATÉ- GIQUE POUR RÉDUIRE LE RISQUE}

Les partenaires qui ont piloté l'étude ont décidé de changer d'approche. Ils en viennent à préconiser qu'une stratégie 
qui se cantonnerait à ne réaliser que des travaux sur les levées ou un nouvel ouvrage écrêteur porterait en elle-même les germes de son propre échec à moyen terme.

Le système de défense des vals de Loire reste en effet toujours vulnérable, en particulier vis-à-vis des brèches accidentelles, surtout s'il n'est pas régulièrement entretenu. Une diminution des dommages qui soit à la hauteur de l'augmentation considérable des enjeux que l'on constate, ne peut reposer uniquement sur une diminution de l'aléa hydraulique. Il faut aujourd'hui prévoir en priorité une diminution de la "vulnérabilité aux inondations" des biens exposés. Ce type de démarche nouvelle relève d'une approche sociale et économique et d'une réglementation harmonieuse du mode de vie en zone inondable.

Toute l'analyse menée sur les aspects hydrauliques, économiques mais aussi sociologiques conduit à décider de la nécessité de ne pas se focaliser uniquement sur des nouveaux aménagements mais aussi de :

- restaurer une culture du risque,

- renforcer notre capacité à gérer au mieux la crue et la post-crue,

pour éviter d'aggraver dramatiquement les conséquences des crues et les dommages, qu'ils apparaissent de manière directe (l'eau atteint une maison ou une entreprise) ou indirecte (arrêt d'exploitation, rupture des communications et des approvisionnements y compris pour des entreprises en dehors de la zone inondable).

\subsection{Accompagner un mode de développement durable dans les vals}

Pour obtenir un bénéfice, dans la durée, de tout autre aménagement, on a proposé de conduire en priorité et en toute hypothèse :

- une stratégie de développement durable des zones inondables,

- un entretien, régulier et dans la durée, du lit du fleuve et du système de protection,

- une excellente capacité de prévision et de prévention de la crue mais aussi de gestion de la crise et de la post-crise.

Cela est déterminant pour assurer l'efficacité des autres mesures envisagées en complément et pour minimiser les dommages directs et indirects, au cours de la crue et lors de la décrue.

La nouveauté réside essentiellement dans la notion de développement durable, qui comprendrait plusieurs actions très concrètes :

- la reconstitution et la diffusion active d'une culture du risque, par une information large des Ligériens et une formation des responsables ayant à intervenir en période de crue : elle utiliserait tous les moyens actuels d'élaboration et de transmission de l'information que sont les médias écrits, radiophoniques ou télévisuels, les jeux de société, les mises en scène de sites touristiques ou paysagers, les interventions dans les écoles, le média Internet, etc.

- la définition d'un mode de développement durable des collectivités locales situées en zone inondable, compatible avec le risque d'inondation et le contrôle de l'urbanisation en zone inondable : cela consistera à trouver les moyens pour que les communes inondables ne voient pas les entreprises et la population qui y résident déserter la zone inondable, les privant ainsi de ressources fiscales et qu'en parallèle elles trouvent un art de vivre et de se développer qui ne consiste pas systématiquement à installer de nouvelles maisons ou de nouvelles entreprises en zone inondable. Une étude pilote conduite auprès d'une vingtaine de communes situées dans deux cantons en amont d'Orléans a jeté les premières bases de solutions pratiques et de leur mise en œuvre expérimentale.

- la diminution de la vulnérabilité des biens exposés aux inondations, pour faire en sorte que l'inondation, qu'on sait un jour inévitable, d'une entreprise installée en zone inondable, coûte le moins cher possible parce qu'on aura profité de toutes ses occasions d'évolution technologique pour rendre les machines mais aussi les modes de travail (gestion des flux, des stocks, relation avec la clientèle, trésorerie, assurances, etc.) moins vulnérables à l'inondation. Des entreprises conscientes du risque se sont montrées intéressées pour conduire ce travail de manière expérimentale. Des groupes de travail associant élus des communes, représentants de l'Etat, représentants des entreprises devraient se mettre en place dans trois lieux différents de la Loire moyenne pour proposer des solutions concrètes dans les mois à venir.

La stratégie devra tenir compte du cadre et de l'échelle territoriales dans lesquels elle se déroulera. Une évolution vers des intercommunalités plus fortes entre communes des vals et des plateaux, serait un atout indéniable. Les orientations agricoles influenceront la nature et la vulnérabilité de l'agriculture. Il semble aussi important de pouvoir mieux maîtriser les réserves foncières qui restent disponibles dans les communes situées en zone inondable, pour aider les élus à élaborer des projets de développement durable, sur des bases solides.

\subsection{Prévention, prévision et gestion de crise}

La prévention est un volet important de la nouvelle stratégie. A la suite de cette étude, on ne pourra plus dire qu'on ne connaissait pas les risques encourus ! Les risques d'aggravation dus à un insuffisant entretien du lit de la Loire sont à prendre en compte dans la durée. De même, il convient de mieux savoir gérer la crise, et surtout la postcrise qui correspond à la décrue. La décrue s'avère bien être la période de tous les dangers, pendant laquelle la négligence et une organisation inadaptée augmenteront beaucoup les dommages.

L'entretien régulier et homogène du lit et du système de protection par levées et déversoirs est un défi important laissé aux Ligériens. En effet, son coût annuel est estimé à près de 15 à $20 \mathrm{MF}$ pour les $450 \mathrm{~km}$ de long et il a été difficile, jusqu'à récemment, d'en conserver les montants sur de longues périodes.

Le modèle hydraulique a permis de bien localiser les sites où un lit insuffisamment entretenu entraîne des surélévations locales de la ligne d'eau de 30 à $70 \mathrm{~cm}$, en particulier dans les agglomérations. Ainsi on a détecté 60 sites prioritaires, représentant $130 \mathrm{~km}$ du lit de la Loire et on peut évaluer, pour l'aval, les répercussions négatives des travaux réalisés à l'amont et donc les prévenir.

En parallèle, l'étude a été l'occasion de définir les modalités d'une intervention soucieuse de la qualité écologique et paysagère du cours d'eau. Pour ce faire, il a fallu dresser l'inventaire de la diversité écologique des milieux, étudier leur dynamique et chercher les modes de gestion écologique compatibles avec le maintien, voire l'enrichissement de cette diversité. 
Le renforcement de la prévention propose de revoir les procédures d'annonce et d'alerte de crue, en fonction des nouvelles informations provenant à la fois du modèle hydraulique et de la connaissance de la répartition des enjeux économiques et sociaux. Il favorisera et accompagnera l'élaboration des dossiers d'information communale sur les risques majeurs prévus par la loi de 1987, ainsi que des dossiers de secours. Cela s'appuiera sur le réseau de collecte et de traitement des données pluviométriques et hydrologiques que la Loire possède de manière pilote depuis 1984 et qui permet de bien anticiper l'apparition des crues (réseau CRISTAL). Les chinois et les polonais profitent actuellement de ce savoir-faire pour constituer des réseaux similaires.

Le modèle hydraulique et la base de données sur les enjeux permettent aujourd'hui de mieux prévoir et anticiper l'inondation des enjeux sensibles : voies d'accès, industries, ressources utiles pour les secours, etc. On peut préparer la gestion de la crise, avec des exercices organisés à l'échelle du bassin.

Une fois la décrue entamée, des entreprises et des équipements sensibles justifient une intervention prioritaire programmée, pour éviter d'augmenter les atteintes et les dommages. Suivant que l'on intervient quelques heures ou quelques jours après le départ de l'eau, les dommages peuvent tripler ou peut-être décupler dans certaines entreprises sensibles. Il faut donc organiser la post-crise et l'assistance auprès de sites prioritaires qui demanderont tous une intervention au même moment. La décrue représente une période à haut risque (fragilité des levées suite à la vidange rapide, fatigue des équipes de surveillance, relâchement de la vigilance, risque de retour trop précoce d'habitants ou d'employés dans les vals inondables en cours de décrue, pollution par piégeage). Là encore, il convient d'anticiper le phénomène et les risques qu'il présente.

Toutes ces actions représentent un investissement de l'ordre de 500 à $700 \mathrm{MF}$ ( 80 à $100 \mathrm{M}$ euros) et un fonctionnement annuel de l'ordre de 25 à $30 \mathrm{MF}$. Cela permet d'assurer la surveillance et l'entretien annuel de tous les ouvrages de protection existants ou à venir, et de disposer du personnel humain qualifié et entraîné pour former les responsables ligériens qui devront intervenir en période de crue (services de l'Etat, élus locaux, industriels) et pour reconstituer la culture du risque.

\subsection{Renforcer les protections existantes et réaliser le Veurdre}

La stratégie comprend aussi des aménagements et des travaux, pour renforcer le système de protection actuel et remédier aux dysfonctionnements constatés et pour améliorer la protection avec la réalisation de l'ouvrage écrêteur du Veurdre.

L'amélioration des défaillances ou insuffisances constatées justifie les interventions suivantes :

- Mieux protéger, avec de nouveaux endiguements très localisés ou des murs de quais, des sites fortement inondés pour des crues inférieures à la centennale. Une partie des 20000 habitants et 2000 entreprises présents dans 40 communes concernées serait mieux protégée.

- Homogénéiser le sommet des levées pour éviter des surverses localisées précoces, dans la mesure où cela n'aggrave pas, de manière prévisible, l'inondation d'enjeux particulièrement sensibles situés en aval. Cela concerne $12 \mathrm{~km}$ de sommet de levée soit $2 \%$ du linéaire.

- Renforcer $90 \mathrm{~km}$ de banquettes sur les levées et fiabiliser $3 \mathrm{~km}$ de fusibles de déversoirs.

- Renforcer le pied des levées bordant le lit mineur sur 125 sites et $130 \mathrm{~km}$ de levées concernés.

- Mieux protéger les enjeux situés dans le prolongement des déversoirs venant à fonctionner.

- Réaliser de nouveaux déversoirs de sécurité dans les vals qui n'en sont pas munis.

Ce programme représente un investissement de l'ordre de 1 à $2 \mathrm{MdF}$, répartis comme suit :

\begin{tabular}{|l|r|}
\hline $\begin{array}{l}\text { - Protections localisées des communes } \\
\text { fréquemment et fortement inondées le } \\
\text { long du cours du fleuve ou dans le } \\
\text { prolongement des déversoirs }\end{array}$ & 300 à $700 \mathrm{MF}$ \\
\hline $\begin{array}{l}\text { - Homogénéisation des levées, } \\
\text { banquettes et fusibles }\end{array}$ & 100 à $150 \mathrm{MF}$ \\
\hline $\begin{array}{l}\text { - Renforcement du pied des levées } \\
\text { bordant le lit mineur }\end{array}$ & $\begin{array}{r}500 \mathrm{MF} \\
\text { à } 1 \mathrm{MdF}\end{array}$ \\
\hline - Nouveaux déversoirs de sécurité & 75 à $150 \mathrm{MF}$ \\
\hline
\end{tabular}

L'ouvrage écrêteur du Veurdre apporte un bénéfice appréciable :

- Il écrête la crue au bec d'Allier de $600 \mathrm{~m}^{3} / \mathrm{s}$ en crue cinquantennale à $900 \mathrm{~m}^{3} / \mathrm{s}$ en crue millénale.

- Il abaisse la cote maximale en Loire de l'ordre de 20 à $50 \mathrm{~cm}$ en amont de Tours et de 5 à $20 \mathrm{~cm}$ en aval, en particulier dans la traversée des agglomérations où le lit est particulièrement resserré. Il repousse à des crues plus rares les débordements et les surverses ; il ne les supprime pas.

- En aval du Bec de Vienne, son effet écrêteur provient d'abord du décalage qu'il induit entre le passage des maximums de la crue de la Loire et de celle de la Vienne.

- Il apporte un bénéfice négligeable à la lutte contre l'apparition des brèches accidentelles, dans la mesure où il n'abaisse pas les vitesses d'écoulement dans le lit mineur et laisse s'écouler en Loire des hauteurs d'eau importantes.

- Il améliore la prévision de la crue et gestion de la crise, en permettant plus facilement de détecter et mesurer le maximum de la crue en aval immédiat du bec d'Allier, en diminuant les niveaux d'eau élevés et donc les longueurs de banquettes à surveiller en crue, en évitant généralement la formation de pics de crue pointus qui aggravent les conditions d'inondation.

L'ouvrage est constitué d'une digue en terre de $1 \mathrm{~km}$ de long et d'une hauteur maximale de $15,5 \mathrm{~m}$ au-dessus du lit mineur. 8 vannes mobiles de $22 \mathrm{~m}$ de large et $5,50 \mathrm{~m}$ de haut chacune peuvent obstruer le lit et permettre le stockage temporaire d'un maximum de $120 \mathrm{Mm}^{3}$ sur une surface de 2400 ha.

C'est un ouvrage respectueux de l'environnement, puisqu'à longueur d'année le seuil est immergé et dispose d'un dispositif favorisant le passage des poissons migrateurs. Une étude a montré que l'ouvrage n'avait aucune influence sensible sur le transport solide du fleuve. 


\section{$\mathrm{V} \square$ CONCLUSION}

Au-delà de l'aménagement hydraulique de la Loire sur lequel les politiques de protection se sont focalisées depuis le siècle dernier, la nouvelle stratégie proposée repose avant tout sur la recherche d'un mode de développement durable des vals. Elle le prévoit compatible avec les crues et les inondations et privilégiant la prévention, la prévision et la gestion de l'événement grave que représentera une crue forte, ainsi que la réduction de la vulnérabilité des biens exposés et la reconstitution d'une culture du risque. Elle rejoint ainsi la résolution du Schéma Directeur d'Aménagement et de Gestion des Eaux du bassin Loire-Bretagne, adopté en 1996, qui préconise de "savoir mieux vivre avec les crues".
La définition des éléments de cette stratégie s'est faite intégralement dans le cadre d'une démarche qui a été dès le départ partenariale entre l'Etat, les collectivités territoriales et l'Agence de l'Eau. Le référent technique partagé que représente l'Equipe pluridisciplinaire s'est avéré un facteur de réussite important, car ses travaux ont été validés en commun, au fur et à mesure de l'avancement des travaux.

La démarche a fait l'objet d'une information régulière et précise, de l'ensemble des acteurs concernés. Celle-ci a révélé une attente importante d'information claire et compréhensible, sur des sujets graves qui préoccupent les responsables ligériens. Cette information a beaucoup participé à faciliter l'adhésion de tous à la démarche, en montrant le sérieux et la transparence avec laquelle les travaux étaient menés. 